\title{
High Performance Molecular Imaging with MALDI Trapped Ion Mobility Time-of-Flight (timsTOF) Mass Spectrometry.
}

\author{
MALDI Trapped Ion Mobility Spectrometry for High Performance Molecular Imaging.
}

Jeffrey M. Spraggins ${ }^{1,2,3^{*}}$, Katerina V. Djambazova ${ }^{2,3}$, Emilio S. Rivera ${ }^{1,3}$, Lukasz G. Migas ${ }^{4}$, Elizabeth K. Neumann ${ }^{1,3}$, Arne Fuetterer ${ }^{5}$, Juergen Suetering ${ }^{5}$, Niels Goedecke ${ }^{5}$, Alice Ly ${ }^{5}$, Raf Van de Plas ${ }^{1,3,4}$ and Richard M. Caprioli ${ }^{1,2,3,6,7}$

${ }^{1}$ Department of Biochemistry, Vanderbilt University, 607 Light Hall, Nashville, TN 37205, USA
2Department of Chemistry, Vanderbilt University, 7330 Stevenson Center, Station B 351822, Nashville,
${ }^{3}$ Mass Spectrometry Research Center, Vanderbilt University, $46521^{\text {st }}$ Ave S \#9160, Nashville, TN 37235,
${ }^{4}$ Delft Center for Systems and Control (DCSC), Delft University of Technology, 2628 CD Delft, The Nethe
${ }^{5}$ Bruker Daltonik GmbH, Fahrenheitstraße 4, 28359 Bremen, Germany
${ }^{6}$ Department of Pharmacology, Vanderbilt University, 2220 Pierce Avenue, Nashville, TN 37232, USA
${ }^{7}$ Department of Medicine, Vanderbilt University, $46521^{\text {st }}$ Ave S \#9160, Nashville, TN 37235, USA

KEYWORDS. Imaging mass spectrometry, Matrix-assisted laser desorption/ionization, Trapped ion mobility spectrometry, timsTOF, Ion mobility mass spectrometry, High spatial resolution imaging, High-throughput imaging, Molecular histology

\begin{abstract}
Imaging mass spectrometry (IMS) enables the spatially targeted molecular assessment of biological tissues at cellular resolutions. New developments and technologies are essential for uncovering the molecular drivers of native physiological function and disease. Instrumentation must maximize spatial resolution, throughput, sensitivity, and specificity, because tissue imaging experiments consist of thousands to millions of pixels. Here, we report the development and application of a matrix-assisted laser desorption/ionization (MALDI) trapped ion mobility spectrometry imaging platform. This prototype MALDI timsTOF instrument is capable of $10 \mu \mathrm{m}$ spatial resolutions and 20 pixels/s throughput molecular imaging. The MALDI source utilizes a Bruker SmartBeam 3-D laser system that can generate a square burn pattern of $<10 \times 10 \mu \mathrm{m}$ at the sample surface. General image performance was assessed using murine kidney and brain tissues and demonstrate that high spatial resolution imaging data can be generated rapidly with mass measurement errors $<5 \mathrm{ppm}$ and $\sim 40,000$ resolving power. Initial TIMS-based imaging experiments were performed on whole body mouse pup tissue demonstrating the separation of closely isobaric $[\mathrm{PC}(32: 0)+\mathrm{Na}]^{+}$and $[\mathrm{PC}(34: 3)+\mathrm{H}]^{+}(3 \mathrm{mDa}$ mass difference) in the gas-phase. We have shown that the MALDI timsTOF platform can maintain reasonable data acquisition rates ( $>2$ pixels/s) while providing the specificity necessary to differentiate components in complex mixtures of lipid adducts. The combination of high spatial resolution and throughput imaging capabilities with high-performance TIMS separations provides a uniquely tunable platform to address many challenges associated with advanced molecular imaging applications.
\end{abstract}

Matrix-assisted laser desorption/ionization (MALDI) imaging mass spectrometry (IMS) combines the sensitivity and molecular specificity of MS with the spatial fidelity of classical microscopy ${ }^{1}$. IMS is routinely used to study a variety of analyte classes including pharmaceuticals ${ }^{2-3}$, metabolites ${ }^{4}$, lipids ${ }^{5}$, nucleic acids, glycans, ${ }^{6-7}$ peptides $^{8-9}$ and proteins ${ }^{10-11}$. In brief, samples are thinly sectioned, mounted onto conductive glass slides, and coated with a light absorbing matrix that assists in desorption and ionization of endogenous molecules. Ions are generated by laser irradiation at each position (pixel) within a designated region of the tissue resulting in the generation of a mass spectrum for each pixel. The spatial resolution of the image is defined by the size of the laser spot at the sample surface, the spacing between pixels in the array (i.e. pitch), and sample preparation processes. A specific molecular image is then generated for each $\mathrm{m} / \mathrm{z}$ by plotting the intensity for the entire array of pixels. The thousands of ion images produced by a single IMS experiment can be compared to histologically stained sections providing molecular context to histological analysis. ${ }^{12}$

The defining performance characteristic of any image-based technology is the effective spatial resolution. Maximizing the resolution with minimal loss of sensitivity is paramount for understanding molecular processes that occur at cellular and subcellular levels ${ }^{13}$ and has motivated considerable efforts in developing new technologies for improving the spatial resolution of IMS. Although sample stage motor precision and matrix considerations are important, the primary means of improving MALDI spatial resolution is to minimize the laser spot size at the sample surface. For a Gaussian profile laser, the diffractionlimited spot size $\left(D_{s}\right)$ is defined by 


$$
D_{s}=M^{2} \frac{4}{\pi} \lambda \frac{f}{D_{b}} \frac{1}{\cos \theta}
$$

Eq. 1

and is proportional to the beam quality factor $(M)$, laser wavelength $(\lambda)$, focal length of the final focusing lens $(f)$, diameter of the input laser beam $\left(D_{b}\right)$, and incident angle of the laser at the sample surface $(\theta)$. Optimizing at least one of these parameters through the integration of pinholes, ${ }^{14}$ optical fibers, ${ }^{15}$ beam expanders, ${ }^{15}$ multiple focusing lenses, coaxial laser illumination, ${ }^{16}$ and transmission geometry optics ${ }^{17}$ results in higher spatial resolution IMS images. Typical commercial IMS platforms are limited to 20-30 $\mu \mathrm{m}$ spatial resolution ${ }^{18}$, while advanced optical systems can achieve 5-10 $\mu \mathrm{m}$ spot sizes using front-side geometries and $>1 \mu \mathrm{m}$ using transmission geometry MALDI setups. ${ }^{15}$ $19-20$

IMS data acquisition throughput becomes critical when spatial resolution improves and a higher numbers of pixels are required. One approach to improve throughput is continuous raster sampling where the laser is fired continuously across the sample surface by moving the stage at a constant velocity. Although the exact location of each pixel can be difficult to determine, pixel size is approximated by accounting for the laser repetition rate, stage velocity, and number of laser shots at a pixel position. This approach can yield data acquisition rates of about 30-100 px/s. ${ }^{21-23}$ Alternatively, the Bruker SmartBeam 3-D laser system achieves high throughput acquisition rates (50-60 px/s) while maintaining discrete pixels by incorporating galvo mirrors into the laser path. The additional mirrors allow laser redirection so that a defined number of laser shots can be collected from a single pixel location. Using this beam steering approach, the laser raster can be performed to effectively collect data from a square pixel with an area set by the operator. ${ }^{24}$

A significant challenge for any IMS experiment is adequately surveying the molecular complexity of tissue and identifying the many hundreds to thousands of chemical species within an individual pixel. Traditionally, chemical identification is performed by either combining high mass accuracy and resolving power to differentiate isobaric species, linking IMS data to offline liquid chromatography (LC)-MS of tissue extracts, ${ }^{25}$ or collecting fragmentation data directly from tissue. High performance mass analyzers (e.g. FT-ICR and Orbitraps) enable high spatial resolution imaging with broad molecular coverage while providing putative identifications by mass accuracy. ${ }^{20,26} \mathrm{How}-$ ever, high mass accuracy alone cannot differentiate structural isomers observed in complex biological samples. By performing parallel IMS and LC-MS experiments, ${ }^{27}$ tandem MS data can be automatically interpreted by pre-existing databases and computational tools. However, LC-MS/MS has limited spatial information and throughput. Direct tissue MS/MS allows high spatial fidelity and tandem MS imaging experiments but is limited by the number of species that can be fragmented from a single pixel in addition to the limited quality of fragment information. ${ }^{28-30}$ Alternatively, ion mobility can be integrated into IMS platforms to enhance specificity, improve molecular coverage, and assist with molecular identification at an acquisition rate compatible with imaging.

Ion mobility-MS provides 2-D separations dependent on the apparent size and shape and mass-to-charge ratio $(\mathrm{m} / \mathrm{z})$ of molecular species. While ion mobility-MS is analogous to LC-MS, gas-phase ion mobility separations are extremely rapid ( $\mu \mathrm{s}-\mathrm{ms}$ ) and completely independent of MALDI ion generation. LC-MS offers enhanced peak capacity over ion mobility-MS, although their lengthy separations (min-hr) make it incompatible with imaging experiments that require acquisition of thousands of pixels. Of particular importance for imaging mass spectrometry experiments, ion mobility-MS enables qualitative identification of chemical class, while suppressing noise for improved sensitivity. ${ }^{31-32}$ Multiple types of ion mobility separations exist, including drift tube ion mobility (DTIM) ${ }^{33-34}$ travelling-wave ion mobility spectrometry (TWIMS), ${ }^{35-36}$ field asymmetric waveform ion mobility spectrometry (FAIMS) ${ }^{37-39}$ and more recently, trapped ion mobility spectrometry (TIMS). ${ }^{40-41}$ The three former techniques have been previously utilized in the imaging domain, studying metabolites,${ }^{42-43}$ lipids,${ }^{44}$ peptides ${ }^{45-46}$ and proteins ${ }^{46-47}$, improving the separation, specificity and identification of species. In recent years, however, TIMS has shown unparalleled resolving power, proving extremely popular in the omics fields. ${ }^{48}$ TIMS separations are capable of resolving powers $>200$ and $>300$ for singly and multiply charged ions, respectively. ${ }^{41,49-52}$ Briefly, ion separation is achieved by using a carrier gas to send ions through a retardant electric field within the TIMS ion guide and is described by:

$$
\Delta K=\frac{v_{g}}{\Delta E_{x}}
$$

Eq. 2

Where the mobility range of the analysis $(\Delta K)$ is proportional to the gas velocity $\left(\mathrm{vg}_{\mathrm{g}}\right)$ and axial electric field gradient $\left(\Delta \mathrm{E}_{\mathrm{x}}\right){ }^{49}$ The electric field gradient (EFG) axially traps and separates ions against the flow of carrier gas as a function of their mobility. Ions are then eluted from the TIMS analyzer by decreasing the retardant electric field. ${ }^{40}$ High-resolution gasphase separations are critical for overcoming the challenges of molecular complexity associated with tissue imaging where LC-based fractionation is not practical.

Here, we report the development and application of a MALDI trapped ion mobility spectrometry orthogonal time-of-flight (timsTOF) imaging platform capable of both standard qTOF MS and TIMS-activated qTOF MS. The development of this system enables high-performance gas-phase separations during image acquisition, enhancing the chemical information that can be acquired from a single tissue section. The MALDI integrated timsTOF combines high spatial resolution and throughput imaging capabilities with increased specificity afforded by TIMS separations.

\section{METHODS}

\section{Materials}

Acetic acid, trifluoroacetic acid (TFA), 1,5-diaminonaphthalene (DAN), ammonium formate, hematoxylin, and eiosin were purchased from Sigma-Aldrich Chemical Co. (St. Louis, MO, USA). HPLC-grade acetonitrile, methanol, ethanol, isopropanol, chloroform were purchased from Fisher Scientific (Pittsburgh, PA, USA).

\section{Sample Preparation:}

A one-week old C57BL/6 control mouse was snapped frozen at $-80^{\circ} \mathrm{C}$, shaved over dry ice, cryosectioned at a $20 \mu \mathrm{m}$ thickness using a CM3050 S cryostat (Leica Biosystems, Wetzlar, Germany), and thaw mounted onto conductive indium tin-oxide coated glass slides (Delta Technologies, Loveland, CO, USA). Rat kidney tissue (Pel-Freeze Biologicals, Rogers, AR, USA) was sectioned at $10 \mu \mathrm{m}$ thickness and thaw-mounted onto a conductive ITO slide. Autofluorescence microscopy images were acquired using EGFP, DAPI and DsRed filters on a Zeiss AxioScan Z1 slide scanner (Carl Zeiss Microscopy GmbH, Oberkochen, Germany) prior to matrix application. DAN matrix was sublimated onto the tissue surface $\left(\sim 1.0 \mathrm{mg} / \mathrm{cm}^{2}\right) .{ }^{53}$ Briefly, 


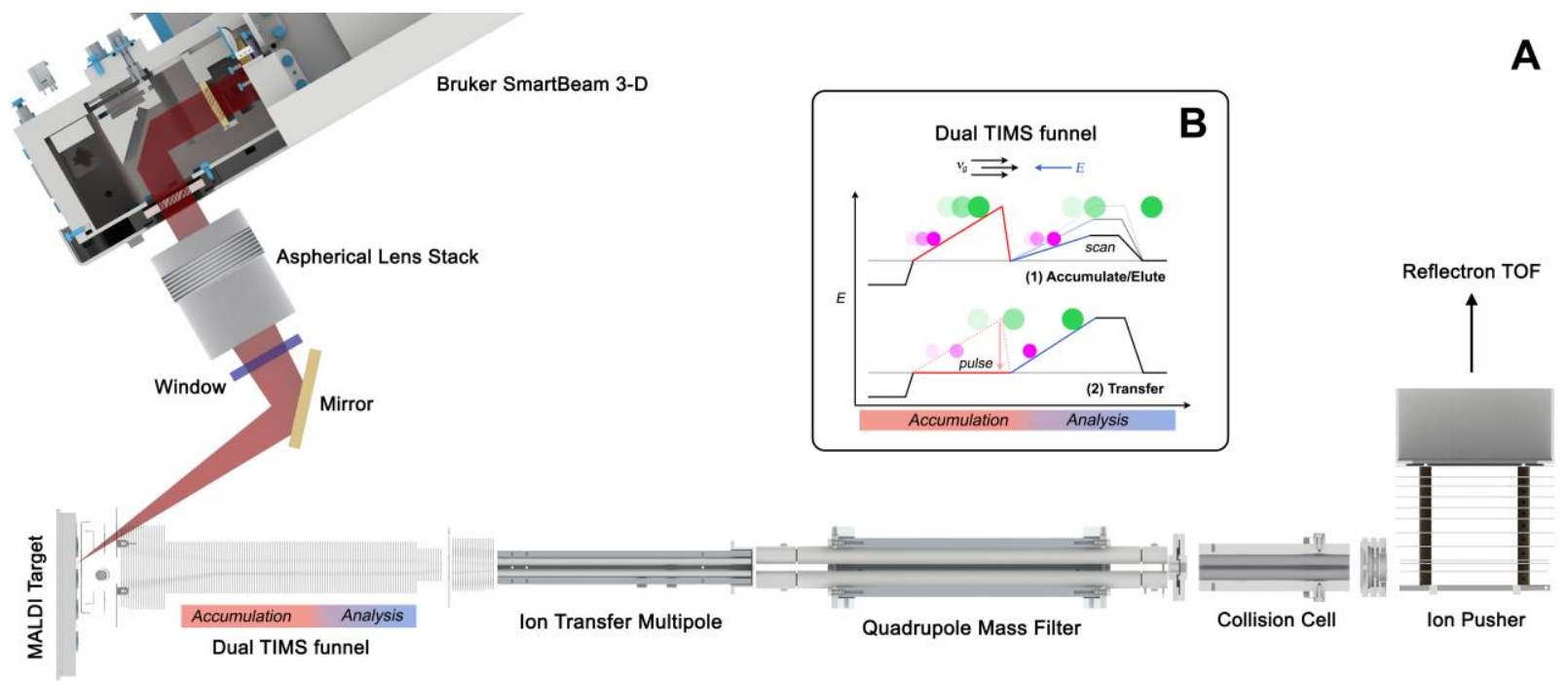

Figure 1. A prototype MALDI timsTOF mass spectrometer. (A) The developed imaging platform couples a MALDI source capable of high spatial resolution and throughput imaging with an ESI timsTOF Pro. (B) Ion mobility separations are performed in a dual TIMS funnel in the first vacuum stage of the instrument. TIMS resolution is tunable by adjusting the EFG scan time and throughput is maximized by performing accumulation and analysis in parallel on sequential pixels.

slides containing tissue were taped to the bottom of the condenser in a simple sublimation apparatus. Approximately $500 \mathrm{mg}$ of DAN was sublimed at $130^{\circ} \mathrm{C}$ and 24 mTorr for $3.5 \mathrm{~min}$.

\section{MALDI timsTOF IMS:}

The TIMS-based MALDI imaging platform was developed from an electrospray ionization (ESI) timsTOF Pro (Bruker Daltonik, Bremen, Germany) and is depicted in Figure 1. The instrument is equipped with a dual ESI/MALDI source with a SmartBeam 3D $10 \mathrm{kHz}$ frequency tripled Nd:YAG laser $(355$ $\mathrm{nm})$. The timsTOF Pro is equipped with a dual TIMS funnel which can perform accumulation and analysis in parallel. The system can be operated in 'qTOF' mode (TIMS deactivated) or utilizing TIMS for performing gas-phase separations during image acquisition. ESI of Agilent ESI-L tunemix was used to perform calibration of $\mathrm{m} / \mathrm{z}$ and ion mobility values. MALDI parameters in both qTOF and TIMS modes were optimized to maximize intensity by tuning ion optics, laser intensity, and laser focus. Additional experimental values include: a ion transfer time of $80 \mu \mathrm{s}$, a pre-pulse storage time of $10 \mu \mathrm{s}$, a collisionRF of $2000 \mathrm{Vpp}$, a TIMS funnel 1 (accumulation)RF of $350 \mathrm{Vpp}$, a TIMS funnel $2 \mathrm{RF}$ (analysis) of $400 \mathrm{Vpp}$, a multipole RF of 500 $\mathrm{Vpp}$, and a collision cell entrance (in) voltage of $300 \mathrm{~V}$.

For ion mobility experiments, TIMS is performed in the first vacuum stage of the mass spectrometer within an augmented ion funnel consisting of an entrance funnel, ion tunnel, and exit funnel. A tailored axial electric field gradient (EFG) is applied to the ion tunnel to trap ions with different mobilities $(\Delta K)$. Following the ion trapping event, the EFG is gradually reduced to allow sequential elution of trapped ions with ascending mobilities. Elongating the ion tunnel enables simultaneous ion accumulation and analysis, maximizing the duty cycle of the experiment (Fig. 1). Further instrument details are provided in the Results and Discussion.

Images were acquired in both qTOF and TIMS modes of operation. qTOF mode images were collected at $10 \mu \mathrm{m}$ and $15 \mu \mathrm{m}$ spatial resolution with beam scan on using 400 laser shots per pixel and $30 \%$ and $35 \%$ laser power respectively. Data were collected in both positive and negative ion modes from $\mathrm{m} / \mathrm{z} 200$ - 1,500. Imaging data collected with TIMS activated were acquired at $20 \mu \mathrm{m}$ spatial resolution with beam scan "on" using 400 laser shots per pixel and $42 \%$ laser power. The TIMS EFG scan time was set to $400 \mathrm{~ms}$. To assess laser characteristics, pixel arrays were generated on sublimated DAN surfaces. With beam scan "off", IMS was performed using 500 laser shots per pixel, 30\% laser energy, and a $10 \mu \mathrm{m}$ pitch. To better visualize the laser raster pattern, the pitch was increased to $30 \mu \mathrm{m}$ with the beam scan activated with 500 laser shots per pixel, $30 \%$ laser energy, and beam scan set to $10 \mu \mathrm{m}$. Burn patterns were measured using the Nikon Eclipse 90i microscope (Nikon Instruments Inc., Melville, NY). All qTOF mode imaging data were visualized using SCiLS Lab Version 2019 (Bruker Daltonics, Bremen, Germany) and TIMS imaging data were analyzed using custom in-house developed software. All lipid identifications were determined based on mass accuracy using the LIPIDMAPS lipidomics gateway (lipidmaps.org) ${ }^{54-55}$ or by performing MS/MS in the collision cell of the protoype MALDI timsTOF platform (40V CID).

\section{MALDI FT-ICR IMS:}

To assist in molecular identification, high mass resolution imaging mass spectrometry of whole-body mouse pup tissue was performed using a 15T MALDI FT-ICR mass spectrometer (Bruker Daltonik, Billerica, MA, USA). The instrument is equipped with an Apollo II dual MALDI/ESI ion source and a 
Smartbeam II 2kHz frequency tripled Nd:YAG laser (355nm). Images were generated using the small laser setting $(\sim 50 \mu \mathrm{m})$ with a pixel spacing of $150 \mu \mathrm{m}$ in both $\mathrm{x}$ and y dimensions. Data was collected with 300 laser shots per pixel at $46 \%$ power from $m / z 460-1,500$ with a data size of $4 \mathrm{MB}$ per spectrum (transient length: $5.2 \mathrm{~s}$ ) resulting in a resolving power of $\sim 600,000$ at $\mathrm{m} / z$ 760 .

\section{Data processing:}

The mouse pup data was exported into a custom binary file format optimized for storage and analysis of ion mobility-IMS data. Each individual spectrum contains between 10,000100,000 centroid peaks that span the acquisition range of $\mathrm{m} / \mathrm{z}$ $300-1200$ that consists of 221,888 bins. The processing pipeline requires common $\mathrm{m} / \mathrm{z}$ axis and centroided peaks that were inserted at their appropriate bin positions while the missing values were set to zero. In addition, each bin has 4,001 ion mobility bins. Following pre-processing, a mean mass spectrum of the entire dataset was generated and peak picked. The 1,000 most intense species were then selected to avoid including chemical noise in the analysis. A mobility profile of each extracted ion was interrogated to identify species with single or multiple conformations.

\section{Histology:}

Immediately following MALDI IMS experiments, matrix was removed from samples using $100 \%$ ethanol and rehydrated with graded ethanol and double distilled $\mathrm{H}_{2} \mathrm{O}$. Tissue was then stained using a hematoxylin and eosin (H\&E) stain. Brightfield microscopy of stained tissues was obtained at 20x magnification using a Leica SCN400 Brightfield Slide Scanner (Leica Microsystems, Buffalo Grove, IL USA).

\section{RESULTS AND DISCUSSION}

\section{Instrumentation Development:}

We have constructed a novel, TIMS-based molecular imaging platform by coupling a MALDI ion source with an ESI timsTOF Pro instrument (Fig. 1). Broadly, this platform provides improved sensitivity, specificity, throughput, and spatial resolution over current commercial platforms. TIMS enables tunable, high-performance, gas-phase separations for differentiation of the many isobaric and isomeric species intrinsically present within biological systems. With respect to the original ESIbased instrument, the source housing has been modified to accommodate a MALDI stage, sample loading chamber, and laser optics. It is designed to load and unload a MALDI sample carrier through a vacuum isolated lock chamber similar to other MALDI platforms. The stage position is controlled by high precision $\mathrm{X}, \mathrm{Y}$ motors, drive screws, slide rails, and linear bearings to provide stage position with a pitch of $5 \mu \mathrm{m}$, while encoders provide positional feedback with micrometer accuracy.

The MALDI source uses a Bruker SmartBeam 3-D laser system, consisting of a frequency tripled solid-state $\mathrm{Nd}$ :YAG laser (wavelength: $355 \mathrm{~nm}$ ) with repetition rates $\leq 10 \mathrm{kHz}$ and internal galvo mirrors that enables beam steering during acquisition. The beam exits the laser as parallel light and is focused by an aspherical lens stack before it enters the vacuum chamber through a window. Inside the vacuum chamber, there is a single flat mirror that directs the beam to the sample surface for generation of ions. The ions are transferred into the mass spectrometer using the TIMS funnel for mass analysis. ESI and MALDI

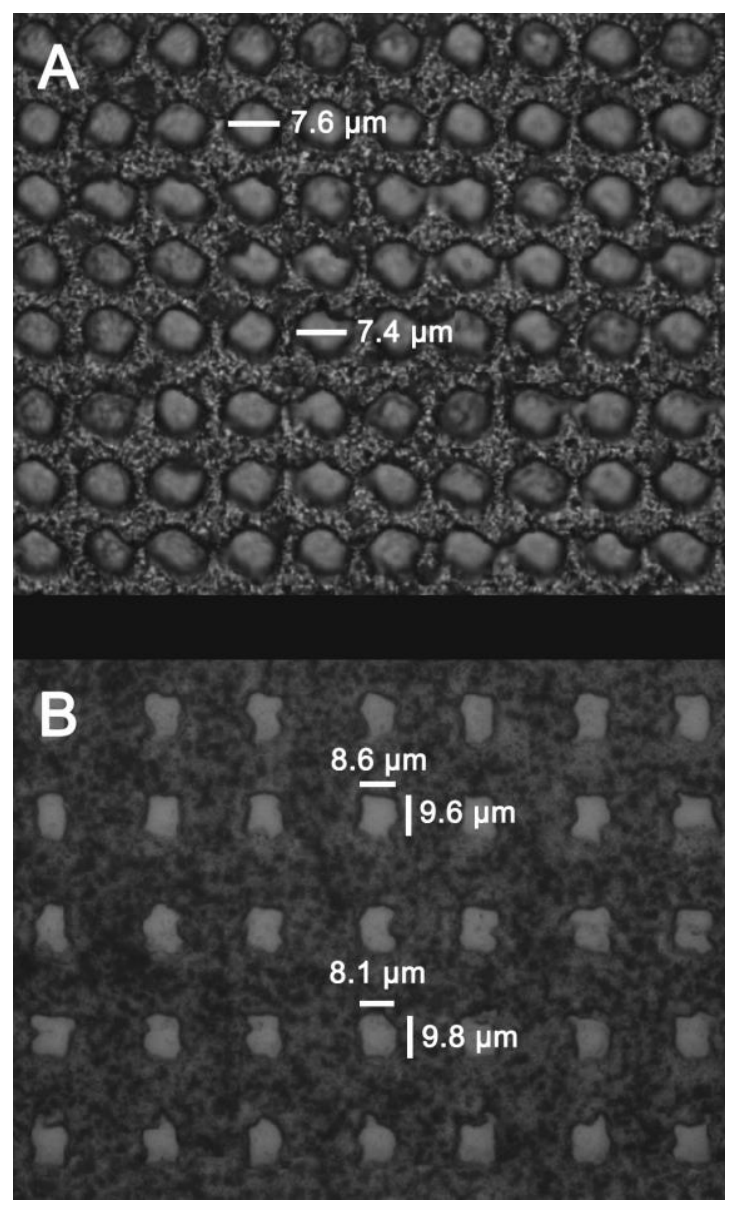

Figure 2. Optical measurements of laser burn patterns generated using the prototype MALDI timsTOF platform. (A) A burn pattern array produced using 500 laser shots, $10 \mu \mathrm{m}$ pitch between pixels, and the beam scan feature turned off showing a beam diameter of $\sim 7.5 \mu \mathrm{m}$ with highly consistent pitch. (B) Burn patterns using 500 laser shots, $30 \mu \mathrm{m}$ pitch and the beam scan set to $10 \mu \mathrm{m}$ showed square-shaped laser ablation areas with an area of $\sim 8.4$ x $9.7 \mu \mathrm{m}$.

experiments can be performed individually or simultaneously without any hardware adjustments.

The system can be operated in qTOF mode by deactivating the ion accumulation and EFG, allowing ions to simply pass through the TIMS funnel. Alternatively, ions can be stored in the accumulation region of the TIMS tunnel and then transferred to the analyzer region for ion mobility separation. Throughput is maximized by accumulating and analyzing ions in parallel, as the TIMS analysis is performed during ion generation and accumulation of ions from the next pixel. Because of the high repetition rate of the laser $(10 \mathrm{kHz})$, the EFG ramp time is typically the limiting factor for pixel acquisition speed. After ions leave the TIMS funnel, they pass through a mass selection quadrupole and collision cell prior to entering the ion pusher and reflection time-of-flight mass analyzer.

\section{High spatial resolution molecular imaging:}

The effective spatial resolution of the MALDI timsTOF platform was assessed by imaging laser burn patterns. Although spot size measurements collected from tissue are expected to be smaller, the high contrast burn patterns of sublimated DAN can 
be more accurately measured. Burn pattern arrays were collected using various laser conditions, such as laser fluence, shot number, and beam scan size, to mimic different imaging experimental setups commonly used for lipid imaging experiments. A burn pattern array was generated with a $10 \mu \mathrm{m}$ pitch between pixels and the beam scan feature turned off to determine the laser spot size at the sample surface (Fig. 2A). Optical measurements of the spot sizes show a beam diameter of $\sim 7.5 \mu \mathrm{m}$ in regular pixel grid with highly consistent pitch. The unperturbed matrix between pixels suggests that $10 \mu \mathrm{m}$ IMS experiments can be performed without oversampling. Instead of an ineffi- cient circular footprint, approximated 'square pixels' can be obtained using beam steering by rastering the laser within the defined area of the pixel creating $\sim 8.4$ x $9.7 \mu \mathrm{m}$ ablation marks (Fig. 2B).

Ablating material and generating ions from square regions maximizes the sampled area during an imaging experiment. For example, circular ablation of a $10 \times 10 \mu \mathrm{m}$ target pixel dimension would sample a $\sim 78.5 \mu \mathrm{m}^{2}$ maximum area (assuming 10 $\mu \mathrm{m}$ diameter). By steering the laser beam to sample a square area, the system can be tuned to ablate material closer to the entire $100 \mu \mathrm{m}^{2}$ pixel area. Assuming a homogenous distribution
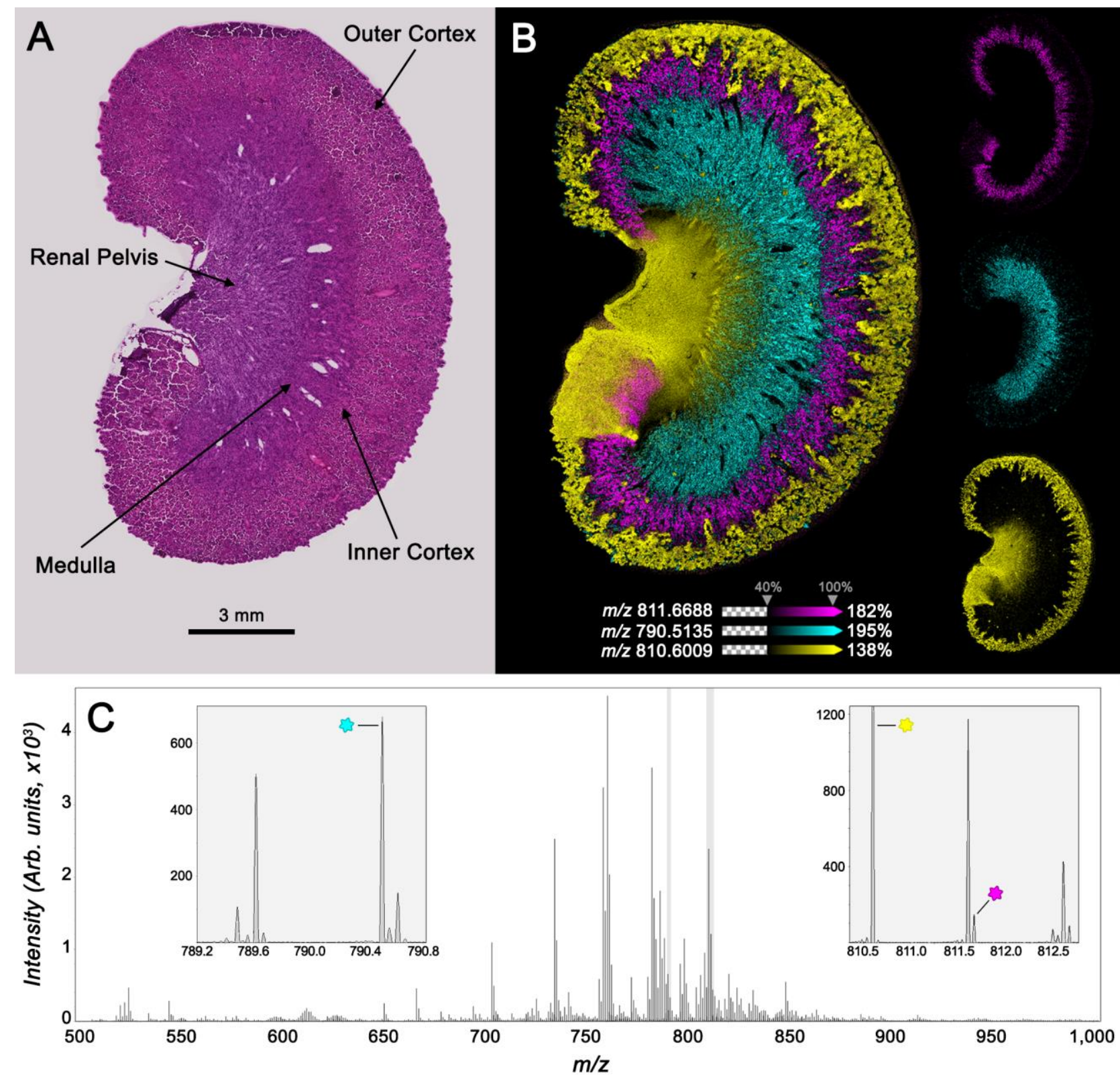

Figure 3. Positive ion mode lipid images collected from rat kidney tissue by MALDI timsTOF IMS operated in qTOF mode. (A) An optical microscopy image of the H\&E stained tissue following IMS analysis shows the major anatomical regions of the tissue. (B) Overlayed and individual images for the selected ions $\mathrm{m} / \mathrm{z} 811.6688\left([\mathrm{SM}(\mathrm{d} 42: 3)+\mathrm{H}]^{+}, 0.12 \mathrm{ppm}\right.$ error, pink), $\mathrm{m} / z .810 .6009$ $\left([\mathrm{PC}(38: 4)+\mathrm{H}]^{+}, 0.25 \mathrm{ppm}\right.$ error, yellow), and $\mathrm{m} / z .790 .5135\left([\mathrm{PE}(\mathrm{P}-38: 4)+\mathrm{K}]^{+},-1.5\right.$ ppm error, cyan) highlight the imaging performance of the platform and the unique distribution of each species. (C) The average spectrum for this experiment shows the spectral performance of the MALDI timsTOF MS and selected $\mathrm{m} / \mathrm{z}$ windows show the multiple isobars detected at each nominal mass. Ions selected for visualization in panel $\mathbf{B}$ are marked (*). 


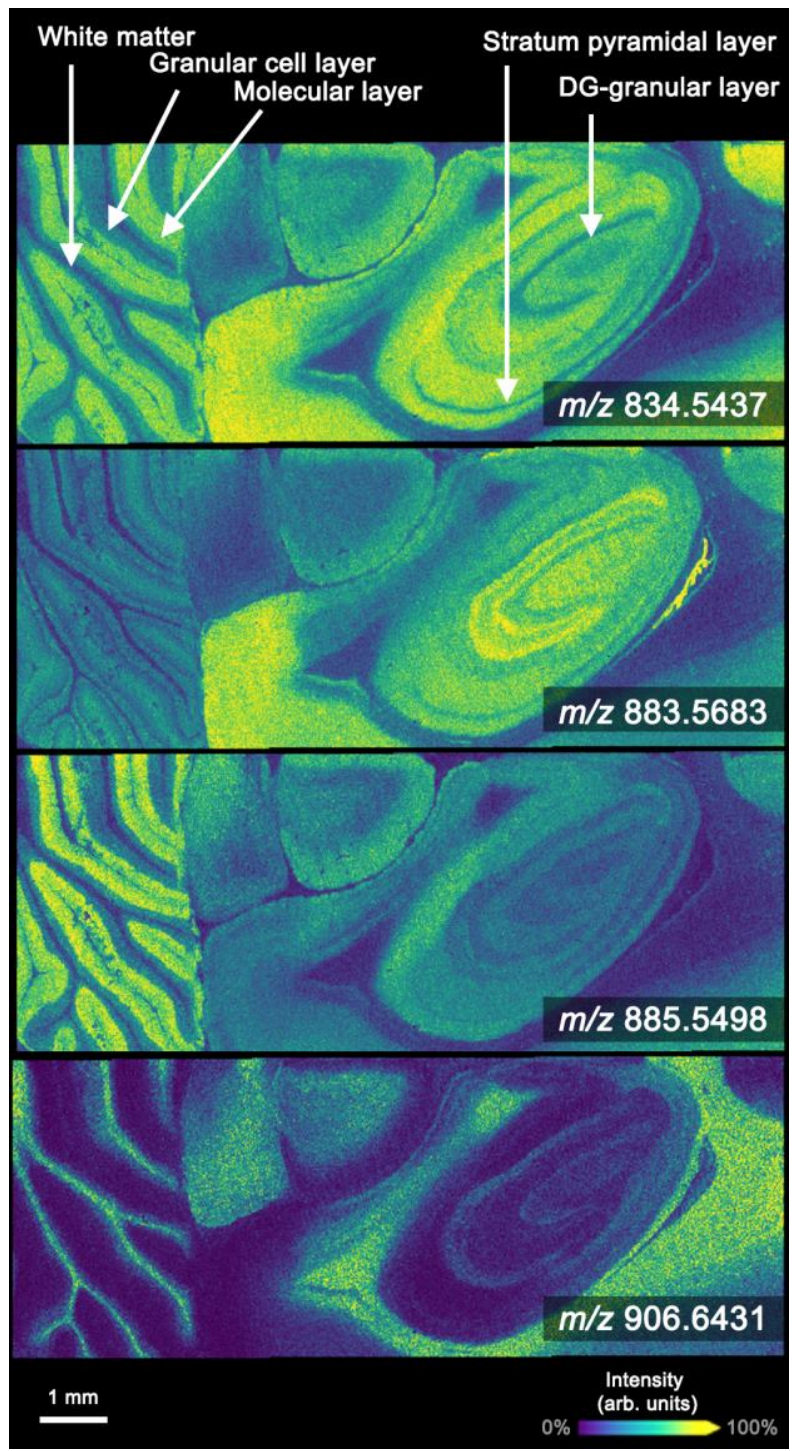

Figure 4. $10 \mu \mathrm{m}$ spatial resolution negative ion mode lipid images collected at $\sim 20$ pixels/s. Data were generated from rat brain tissue by MALDI timsTOF IMS operated in qTOF mode. Ions were selected to show the variety of lipid distributions observed in the experiment: $m / z$ 834.5437 ([PS(40:6)-H]-, -0.12 ppm error), $m / z \quad 883.5683$ ([PI(38:5)-H]', -0.45 ppm error), $m / z 885.5498$ ([PI(38:4)-H] $]^{-},-0.23 \mathrm{ppm}$ error), and $m / z 906.6431$ ([SHex$\operatorname{Cer}(\mathrm{t} 42: 1)-\mathrm{H}]^{-},-1.9 \mathrm{ppm}$ error). The prototype imaging platform is able to resolve the various fine tissue structures of the cerebellum (e.g. white matter, granular cell layer, and molecular layer) and the hippocampus (e.g. stratum pyramidal layer and DGgranular layer).

of molecules across the pixel, square pixels increase the number of sampled molecules by $\sim 27 \%$, resulting in improved sensitivity. Efficient sampling is of particular importance for high spatial resolution experiments because as pixels become smaller the number of molecules sampled decreases and the measurement approaches the limit of detection of the instrument.

Normal, wild-type murine tissues were used to assess the performance of the MALDI TIMS system for molecular imaging in qTOF mode (Fig. 3). Positive ion mode rat kidney lipid images were generated from $\mathrm{m} / \mathrm{z}, 500-1,500$ at $15 \mu \mathrm{m}$ spatial res- olution with the beam scan activated. Imaging data were collected from the entire kidney tissue section including: outer cortex, inner cortex, medulla, and renal pelvis (Fig. 3A). Ions localizing to specific tissue subregions highlight image quality and demonstrate the ability to chemically differentiate the kidney (Fig. 3B). [SM $(\mathrm{d} 42: 3)+\mathrm{H}]^{+}(\mathrm{m} / \mathrm{z} 811.6688,0.12 \mathrm{ppm}$ error $)$, $[\mathrm{PC}(38: 4)+\mathrm{H}]^{+}(\mathrm{m} / z$ 810.6009, $0.25 \mathrm{ppm}$ error $)$, and $[\mathrm{PE}(\mathrm{P}-$ $38: 4)+\mathrm{K}]^{+}(\mathrm{m} / z, 790.5135,-1.5 \mathrm{ppm}$ error) localized to the tubules of the inner cortex, outer cortex and renal pelvis, and medulla, respectively. The average mass spectrum for the entire imaging experiment is shown in Figure 3C. Over $2300 \mathrm{~m} / \mathrm{z}$ peaks were detected with an $\mathrm{S} / \mathrm{N}>10$ demonstrating the overall sensitivity and molecular coverage of the imaging system. The resolving power of the measurements was $\sim 40,000$ at $\mathrm{m} / \mathrm{z} 800$, enabling differentiation of nominally isobaric species.

Negative ion mode images were also generated of the hind brain and hippocampus of transversally sectioned rat brain tissue to demonstrate the high spatial resolution and throughput capabilities of the system (Fig. 4). Fine differentiation of the white matter, granular cell layer, and molecular layer of the cerebellum was achieved. Lipid images show specific localization to the various layers of the hippocampus including the pyramidal cell layer and the granule layer of the dentate gyrus. In this dataset, 249,644 total pixels were acquired in $\sim 3.5$ hours producing an effective data acquisition rate of $\sim 20$ pixels/s. Similar to the rat kidney tissue analysis, data generated from rat brain tissue resulted in a mass measurement error $<2 \mathrm{ppm}$ and a resolving power of $\sim 40,000$ at $\mathrm{m} / \mathrm{z} 800$. Other imaging platforms can achieve similar spectral performance but require longer scan times or can generate data very rapidly but cannot achieve the same spatial or mass resolution.

\section{MALDI TIMS Imaging Mass Spectrometry:}

MALDI TIMS imaging of a whole body mouse pup tissue was used to evaluate the effectiveness of the system. Positive ion mode TIMS lipid images were collected with a $50 \mu \mathrm{m}$ spatial resolution and utilizing a $400 \mathrm{~ms}$ EFG scan time. Ion mobility provides drift time $\left(1 / \mathrm{K}_{0}\right)$ as an additional data dimension to typical imaging data (i.e. $\mathrm{x} / \mathrm{y}$ locations and $\mathrm{m} / \mathrm{z}$ value). Ion mobility information for this system can be interpreted from an average mass spectrum or mobility heat map $\left(\mathrm{m} / \mathrm{z}\right.$ vs. $1 / \mathrm{K}_{0}$, Supplemental Fig. S1). The mobility heat map covers the entire collected $\mathrm{m} / \mathrm{z}$, range for the mouse pup. Mobility decreases with larger $\mathrm{m} / z$, values as relative collisional cross sections are proportionally related to the $m / z$ value of the ion. The spectral data show the characteristic lipid distribution of biological samples with the most intense signals found between $\mathrm{m} / \mathrm{z} 650$ - 900 . A preliminary survey of the data found that, of the 1,000 most intense ions (overall mean spectrum), 289 species were detected that displayed multiple mobilities by TIMS. A prime example of this phenomena are the ions observed at $\mathrm{m} / \mathrm{z}, 756.5517$ (Fig. 5). The extracted ion mobilogram showed at least three different species resolved across the tissue sample and, interestingly, the spatial distributions vary dramatically between the different species. The ion detected at $\mathrm{m} / \mathrm{z} 756.5517$ with a mobility of $1.45-1.48\left(1 / \mathrm{K}_{0}\right)$ was detected throughout the animal but had lower intensity within the brain and brain stem (Fig. $\mathbf{5 A}$ ). Conversely, the distribution of the isobar with the mobility of 1.48 - $1.53\left(1 / \mathrm{K}_{0}\right)$ was abundant within the brain, spine, and intestines (Fig. 5B). However, the image of the average ion intensity covering both species $\left(1 / \mathrm{K}_{0} 1.45-1.53\right)$ is dominated by the more abundant species with a $1 / \mathrm{K}_{0}$ of $1.48-1.53$ (Fig. 5C). These ion images can only be differentiated because of the 

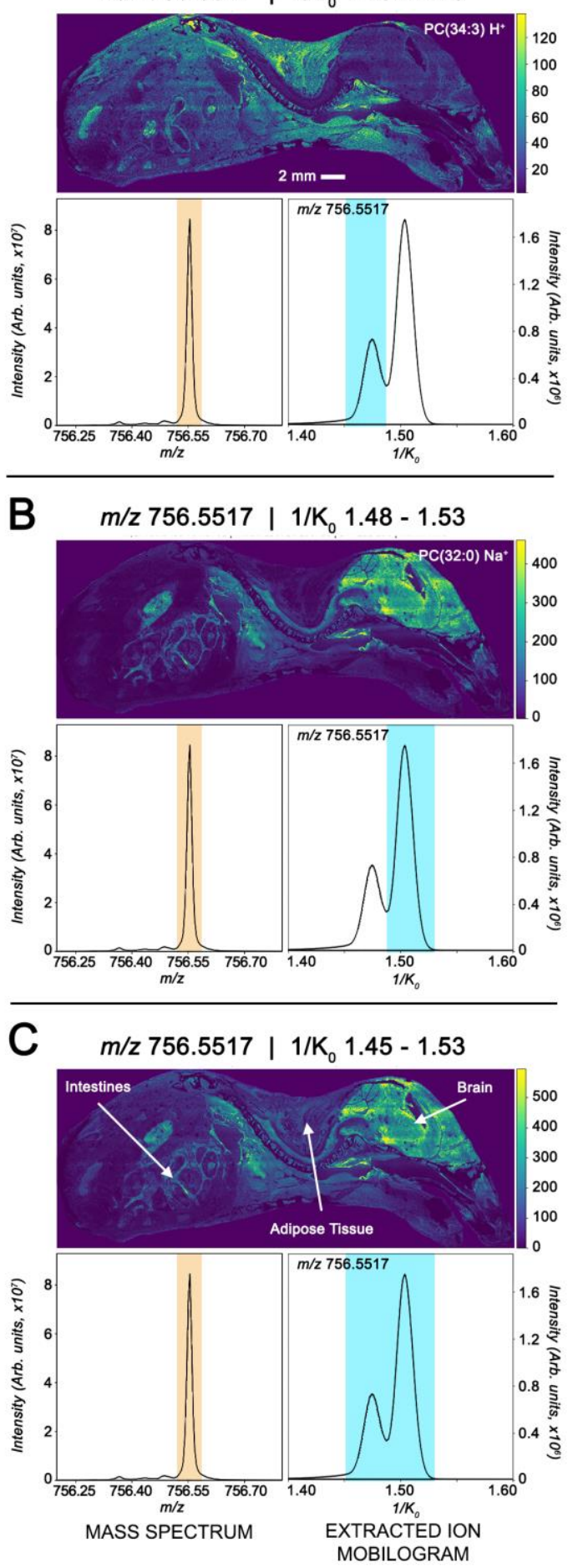

Figure 5. Ion mobility IMS data collected from a whole body mouse pup tissue collected at $50 \mu \mathrm{m}$ spatial resolution and a 400 ms TIMS scan time. (A-C) Each panel highlights the positive ion mode images from selected mobility ranges for $m / z$ 756.5517 and only one peak is detected in the selected mass window (orange) while multiple conformations are observed in the extracted ion mobilogram. (A) $[\mathrm{PC}(34: 3)+\mathrm{H}]^{+}$is found throughout the tissue except the brain and spinal cord. (B) $[\mathrm{PC}(32: 0)+\mathrm{Na}]^{+}$localizes in the brain, spine and intestines. $(\mathbf{C})$ Ion image taken from $1 / \mathrm{K}_{0} 1.45-1.53$, is dominated by the higher intensity $[\mathrm{PC}(32: 0)+\mathrm{Na}]^{+}$mimicking results from a nonion mobility IMS experiment.
TIMS separation and this observation would not be possible using mass analyzers with similar performance without ion mobility. The EFG scan time is tunable allowing the ion mobility resolution to vary based on experimental needs. Although longer scan times $(>500 \mathrm{~ms})$ are generally not compatible with LC-based experiments, imaging experiments provide more flexibility and can accommodate longer scan times. There is a significant loss of ion mobility resolution and information at lower data acquisition rates, demonstrating a need for increasing scan time when possible. Imaging throughput can be adjusted without any reduction in spatial information, easily accommodating longer TIMS analysis times. This is analogous to imaging mass spectrometry performed on FT-ICR or Orbitrap systems where FTMS scan times are tuned to facilitate high mass resolution data acquisition. This example highlights the power of TIMS separations and ion mobility in general for imaging experiments to improve specificity and molecular coverage in direct tissue analysis.

Off-line ultra-high mass resolution MALDI FT-ICR MS and MS/MS were used to identify the species observed at $m / z$ 756.5517 within the above TIMS IMS experiment. A serial whole body mouse pup tissue section was imaged by MALDI FT-ICR MS at $150 \mu \mathrm{m}$ spatial resolution and $\sim 600,000$ resolving power at $\mathrm{m} / \mathrm{z}, 760$, uncovering two nominally isobaric species, $[\mathrm{PC}(32: 0)+\mathrm{Na}]^{+}(\mathrm{m} / \mathrm{z}, 756.55087 ;-0.70 \mathrm{ppm}$ error $)$ and $[\mathrm{PC}(34: 3)+\mathrm{H}]^{+}(\mathrm{m} / \mathrm{z} 756.55325 ;-0.73 \mathrm{ppm}$ error $)$ at $\mathrm{m} / z \mathbf{z} 756.5$ (Supplemental Fig. 2). The spatial distributions of each lipid were used to link the FT-ICR MS data to the MALDI timsTOF images and it was determined that the ion with a mobility of $1.48-1.53$ was $[\mathrm{PC}(32: 0)+\mathrm{Na}]^{+}$while $[\mathrm{PC}(34: 3)+\mathrm{H}]^{+}$was the ion with a $1 / \mathrm{K}_{0}$ value of $1.45-1.48$. This was confirmed by collecting MS/MS data from areas of adipose and brain tissue (Supplemental Fig. 3) in a serial tissue section using the protoype MALDI timsTOF instrument. Although these ions are not isomers, they require resolving powers $>300,000$ at $\mathrm{m} / \mathrm{z}, 760$ to resolve them solely with a mass analyzer. Resolutions of this magnitude are challenging to achieve and often require lengthy acquisition times for any mass analyzer. Even high-field FTMS platforms require long scan times $(\sim 1.5-3 \mathrm{~s})$ for the necessary resolving power, making the experiment impractical for most imaging applications. However, TIMS imaging separated these species while maintaining a throughput $>2 \mathrm{px} / \mathrm{s}$ because these ions have different mobilities. The FT-ICR IMS experiment would require over 200 hours to achieve base-line resolution of these two isobars at the same spatial resolution as acquired on the TIMS imaging experiment, leading to a ten-fold increase in analysis speed. Differentiating various ionizing adducts (e.g. $\mathrm{H}^{+}, \mathrm{Na}^{+}$and $\mathrm{K}^{+}$) is important for imaging experiments as they are regularly generated by MALDI analysis of tissue and cannot be removed without additional sample preparation. Unless MS/MS is performed, differences in the distributions of these species typically goes unnoticed, potentially reducing the quality or accuracy of resulting IMS information.

\section{CONCLUSIONS}

The performance and application of a prototype MALDI timsTOF imaging mass spectrometer that is capable of $10 \mu \mathrm{m}$ spatial resolutions and 20 pixels/s throughput has been demonstrated. The orthogonal time-of-flight mass analyzer is capable of measuring ions with mass measurement errors $<5 \mathrm{ppm}$ and resolutions $\sim 40,000$ while maintaining high-speed data acquisition. Imaging data collected with TIMS improves specificity and molecular coverage for direct tissue analysis compared to non-ion mobility based systems. As a proof-of-concept, it was 
showed that TIMS could separate nominally isobaric $[\mathrm{PC}(32: 0)+\mathrm{Na}]^{+}$and $[\mathrm{PC}(34: 3)+\mathrm{H}]^{+}(\Delta 3 \mathrm{mDa})$ in a fraction of the time required to differentiate them using high mass resolution systems. The unique combination of spatial resolution, throughput, spectral performance, and high-performance gasphase separations is critical for advanced molecular imaging applications, particularly for metabolite and lipid analysis where mass redundancy and structural isomers are prevalent. By precisely defining the ion mobility resolution and pixel size during MALDI timsTOF analysis, it is possible to vary molecular imaging experiments depending on experimental requirements and limitations, allowing for multidimensional specificity (e.g. spatial distribution, $\mathrm{m} / z$, and high-resolution TIMS). The capabilities of the developed TIMS-based platform will provide a critical need to the imaging community by supplementing the lack of chromatographic separation in IMS studies. By broadly improving spatial resolution, speed, sensitivity and specificity, this imaging platform will significantly increase the ability to fully investigate the molecular drivers of biological processes.

\section{ASSOCIATED CONTENT}

Supporting Information

The Supporting Information is available free of charge on the ACS Publications website.

Supplemental Figures showing the MALDI timsTOF IMS average mass spectrum and ion mobility heat map (Fig. S1) and MALDI FT-ICR IMS data from whole body mouse pup tissue (Fig. S2). (PDF)

\section{AUTHOR INFORMATION}

Corresponding Author

*Email: jeff.spraggins@ vanderbilt.edu

\section{ORCID}

Jeffrey M. Spraggins: 0000-0001-9198-5498

Katerina V. Djambazova: 0000-0002-2680-9014

Emilio S. Rivera: 0000-0003-3215-1092

Lukasz G. Migas: 0000-0002-1884-6405

Elizabeth K. Neumann: 0000-0002-6078-3321

Arne Fuetterer: 0000-0003-1550-719X

Juergen Suetering: 0000-0003-0423-2952

Niels Goedecke: 0000-0002-3966-0853

Alice Ly: 0000-0002-0827-6840

Raf Van de Plas: 0000-0002-2232-7130

Richard M. Caprioli: 0000-0001-5859-3310

\section{Author Contributions}

The manuscript was written through the contributions of all authors. All authors have given approval to the final version of the manuscript.

\section{ACKNOWLEDGMENT}

Instrument development was supported by the National Science Foundation Major Research Instrument Program through an instrument development grant awarded to J.M.S. and R.M.C. (CBET 1828299). The 15T FT-ICR MS in the Mass Spectrometry Research Center at Vanderbilt University was acquired through the NIH Shared Instrumentation Grant Program (1S10OD012359). E.K.N. is supported by a National Institute of Environmental Health Sciences training grant (T32ES007028). Further support was provided by the NIH Common Fund and National Institute of Diabetes and Digestive and Kidney Diseases (U54DK120058 awarded to J.M.S. and R.M.C.), the NIH National Institute of Allergy and Infectious Diseases (awarded to J.M.S. and Eric P. Skaar), and by the NIH National Institute of General Medical Sciences (2P41GM103391 awarded to R.M.C.). The authors also wish to thank Jens Hoehndorf and D. Shannon Cornett of Bruker Daltonik for their guidance and technical input as well as Terry Dermody from the University of Pittsburgh for providing the mouse pup and Daniel Ryan for sample preparation assistance.

\section{REFERENCES}

1. Caprioli, R. M.; Farmer, T. B.; Gile, J., Molecular imaging of biological samples: localization of peptides and proteins using MALDI-TOF MS. Anal Chem 1997, 69 (23), 4751-60.

2. Nilsson, A.; Goodwin, R. J.; Shariatgorji, M.; Vallianatou, T.; Webborn, P. J.; Andren, P. E., Mass spectrometry imaging in drug development. Anal Chem 2015, 87 (3), 1437-55.

3. Huang, J. T.; Hannah-Qiuhua, L.; Szyszka, R.; Veselov, V.; Reed, G.; Wang, X.; Price, S.; Alquier, L.; Vas, G., Molecular imaging of drug-eluting coronary stents: method development, optimization and selected applications. J Mass Spectrom 2012, 47 (2), 155-62.

4. $\quad$ Bhandari, D. R.; Schott, M.; Rompp, A.; Vilcinskas, A.; Spengler, B., Metabolite localization by atmospheric pressure highresolution scanning microprobe matrix-assisted laser desorption/ionization mass spectrometry imaging in whole-body sections and individual organs of the rove beetle Paederus riparius. Anal Bioanal Chem 2014.

5. Berry, K. A.; Hankin, J. A.; Barkley, R. M.; Spraggins, J. M.; Caprioli, R. M.; Murphy, R. C., MALDI imaging of lipid biochemistry in tissues by mass spectrometry. Chem Rev 2011, 111 (10), 6491-512.

6. Drake, R. R.; Powers, T. W.; Norris-Caneda, K.; Mehta, A. S.; Angel, P. M., In Situ Imaging of N-Glycans by MALDI Imaging Mass Spectrometry of Fresh or Formalin-Fixed ParaffinEmbedded Tissue. Current Protocols in Protein Science 2018, 94 (1), e68.

7. Angel, P. M.; Mehta, A.; Norris-Caneda, K.; Drake, R. R., MALDI Imaging Mass Spectrometry of N-glycans and Tryptic Peptides from the Same Formalin-Fixed, Paraffin-Embedded Tissue Section. In Tissue Proteomics: Methods and Protocols, Sarwal, M. M.; Sigdel, T. K., Eds. Springer New York: New York, NY, 2018; pp 225-241.

8. Groseclose, M. R.; Andersson, M.; Hardesty, W. M.; Caprioli, R. M., Identification of proteins directly from tissue: in situ tryptic digestions coupled with imaging mass spectrometry. $J$ Mass Spectrom 2007, 42 (2), 254-62.

9. Schober, Y.; Guenther, S.; Spengler, B.; Rompp, A., High-resolution matrix-assisted laser desorption/ionization imaging of tryptic peptides from tissue. Rapid Commun Mass Spectrom 2012, 26 (9), 1141-6.

10. Deutskens, F.; Yang, J.; Caprioli, R. M., High spatial resolution imaging mass spectrometry and classical histology on a single tissue section. J Mass Spectrom 2011, 46 (6), 568-71. 
11.

Seeley, E. H.; Schwamborn, K.; Caprioli, R. M., Imaging of intact tissue sections: moving beyond the microscope. $J$ Biol Chem 2011, 286 (29), 25459-66.

12. El-Aneed, A.; Cohen, A.; Banoub, J., Mass Spectrometry, Review of the Basics: Electrospray, MALDI, and Commonly Used Mass Analyzers. Applied Spectroscopy Reviews 2009, 44 (3), 210-230.

13. Neumann, E. K.; Do, T. D.; Comi, T. J.; Sweedler, J. V., Exploring the Fundamental Structures of Life: Non-Targeted, Chemical Analysis of Single Cells and Subcellular Structures. Angewandte Chemie International Edition 2019, 58 (28), 93489364.

14. Zavalin, A.; Yang, J.; Caprioli, R., Laser Beam Filtration for High Spatial Resolution MALDI Imaging Mass Spectrometry. Journal of The American Society for Mass Spectrometry 2013, 24 (7), 1153-1156.

15. Feenstra, A. D.; Dueñas, M. E.; Lee, Y. J., Five Micron High Resolution MALDI Mass Spectrometry Imaging with Simple, Interchangeable, Multi-Resolution Optical System. Journal of The American Society for Mass Spectrometry 2017, 28 (3), 434-442.

16. Guenther, S.; Koestler, M.; Schulz, O.; Spengler, B., Laser spot size and laser power dependence of ion formation in high resolution MALDI imaging. International Journal of Mass Spectrometry 2010, 294 (1), 7-15.

17. Zavalin, A.; Todd, E. M.; Rawhouser, P. D.; Yang, J.; Norris, J. L.; Caprioli, R. M., Direct imaging of single cells and tissue at sub-cellular spatial resolution using transmission geometry MALDI MS. Journal of Mass Spectrometry 2012, 47 (11), 1473-1481.

18. Norris, J. L.; Caprioli, R. M., Imaging mass spectrometry: A new tool for pathology in a molecular age. PROTEOMICS - Clinical Applications 2013, 7 (11-12), 733-738.

19. Römpp, A.; Spengler, B., Mass spectrometry imaging with high resolution in mass and space. Histochemistry and Cell Biology 2013, 139 (6), 759-783.

20. Kompauer, M.; Heiles, S.; Spengler, B., Atmospheric pressure MALDI mass spectrometry imaging of tissues and cells at 1.4- $\mu \mathrm{m}$ lateral resolution. Nature Methods 2016, 14, 90.

21. Prentice, B. M.; Chumbley, C. W.; Caprioli, R. M., Highspeed MALDI MS/MS imaging mass spectrometry using continuous raster sampling. Journal of Mass Spectrometry 2015, 50 (4), 703-710.

22. Spraggins, J. M.; Caprioli, R. M., High-Speed MALDITOF Imaging Mass Spectrometry: Rapid Ion Image Acquisition and Considerations for Next Generation Instrumentation. Journal of The American Society for Mass Spectrometry 2011, 22 (6), 10221031.

23. Barré, F.; Rocha, B.; Dewez, F.; Towers, M.; Murray, P.; Claude, E.; Cillero-Pastor, B.; Heeren, R.; Porta Siegel, T., Faster raster matrix-assisted laser desorption/ionization mass spectrometry imaging of lipids at high lateral resolution. International Journal of Mass Spectrometry 2019, 437, 38-48.

24. Spraggins, J. M.; Rizzo, D. G.; Moore, J. L.; Noto, M. J.; Skaar, E. P.; Caprioli, R. M., Next-generation technologies for spatial proteomics: Integrating ultra-high speed MALDI-TOF and high mass resolution MALDI FTICR imaging mass spectrometry for protein analysis. PROTEOMICS 2016, 16 (11-12), 1678-1689. 25. Théron, L.; Centeno, D.; Coudy-Gandilhon, C.; PujosGuillot, E.; Astruc, T.; Rémond, D.; Barthelemy, J.-C.; Roche, F.; Feasson, L.; Hébraud, M.; Béchet, D.; Chambon, C., A Proof of Concept to Bridge the Gap between Mass Spectrometry Imaging, Protein Identification and Relative Quantitation: MSI LCMS/MS-LF. Proteomes 2016, 4 (4), 32.

26. Smith, D. F.; Kiss, A.; Leach, F. E.; Robinson, E. W.; Paša-Tolić, L.; Heeren, R. M. A., High mass accuracy and high mass resolving power FT-ICR secondary ion mass spectrometry for biological tissue imaging. Analytical and Bioanalytical Chemistry 2013, 405 (18), 6069-6076.
27. Dilillo, M.; Ait-Belkacem, R.; Esteve, C.; Pellegrini, D.; Nicolardi, S.; Costa, M.; Vannini, E.; Graaf, E. L. d.; Caleo, M.; McDonnell, L. A., Ultra-High Mass Resolution MALDI Imaging Mass Spectrometry of Proteins and Metabolites in a Mouse Model of Glioblastoma. Scientific Reports 2017, 7 (1), 603.

28. Hansen, R. L.; Lee, Y. J., Overlapping MALDI-Mass Spectrometry Imaging for In-Parallel MS and MS/MS Data Acquisition without Sacrificing Spatial Resolution. Journal of The American Society for Mass Spectrometry 2017, 28 (9), 1910-1918. 29. Dueñas, M. E.; Larson, E. A.; Lee, Y. J., Toward Mass Spectrometry Imaging in the Metabolomics Scale: Increasing Metabolic Coverage Through Multiple On-Tissue Chemical Modifications. Frontiers in Plant Science 2019, 10 (860).

30. Kaletaş, B. K.; van der Wiel, I. M.; Stauber, J.; Dekker, L. J.; Güzel, C.; Kros, J. M.; Luider, T. M.; Heeren, R. M. A., Sample preparation issues for tissue imaging by imaging MS. PROTEOMICS 2009, 9 (10), 2622-2633.

31. Huang, J.-T.; Hannah-Qiuhua, L.; Szyszka, R.; Veselov, V.; Reed, G.; Wang, X.; Price, S.; Alquier, L.; Vas, G., Molecular imaging of drug-eluting coronary stents: method development, optimization and selected applications. Journal of Mass Spectrometry 2012, 47 (2), 155-162.

32. Bhandari, D. R.; Schott, M.; Römpp, A.; Vilcinskas, A.; Spengler, B., Metabolite localization by atmospheric pressure highresolution scanning microprobe matrix-assisted laser desorption/ionization mass spectrometry imaging in whole-body sections and individual organs of the rove beetle Paederus riparius. Analytical and Bioanalytical Chemistry 2015, 407 (8), 2189-2201. 33. May, J. C.; Goodwin, C. R.; Lareau, N. M.; Leaptrot, K. L.; Morris, C. B.; Kurulugama, R. T.; Mordehai, A.; Klein, C.; Barry, W.; Darland, E.; Overney, G.; Imatani, K.; Stafford, G. C.; Fjeldsted, J. C.; McLean, J. A., Conformational Ordering of Biomolecules in the Gas Phase: Nitrogen Collision Cross Sections Measured on a Prototype High Resolution Drift Tube Ion MobilityMass Spectrometer. Analytical Chemistry 2014, 86 (4), 2107-2116. 34. Valentine, S. J.; Koeniger, S. L.; Clemmer, D. E., A Split-Field Drift Tube for Separation and Efficient Fragmentation of Biomolecular Ions. Analytical Chemistry 2003, 75 (22), 62026208 .

35. Zhong, Y.; Hyung, S.-J.; Ruotolo, B. T., Characterizing the resolution and accuracy of a second-generation traveling-wave ion mobility separator for biomolecular ions. Analyst 2011, 136 (17), 3534-3541.

36. Shvartsburg, A. A.; Smith, R. D., Fundamentals of Traveling Wave Ion Mobility Spectrometry. Analytical Chemistry 2008, 80 (24), 9689-9699.

37. Purves, R. W.; Guevremont, R., Electrospray Ionization High-Field Asymmetric Waveform Ion Mobility Spectrometry-Mass Spectrometry. Analytical Chemistry 1999, 71 (13), 2346-2357.

38. Kolakowski, B. M.; Mester, Z., Review of applications of high-field asymmetric waveform ion mobility spectrometry (FAIMS) and differential mobility spectrometry (DMS). Analyst 2007, 132 (9), 842-864.

39. Guevremont, R., High-field asymmetric waveform ion mobility spectrometry: A new tool for mass spectrometry. Journal of Chromatography A 2004, 1058 (1), 3-19.

40. Fernandez-Lima, F.; Kaplan, D. A.; Suetering, J.; Park, M. A., Gas-phase separation using a trapped ion mobility spectrometer. International Journal for Ion Mobility Spectrometry 2011, 14 (2), 93-98.

41. Michelmann, K.; Silveira, J. A.; Ridgeway, M. E.; Park, M. A., Fundamentals of Trapped Ion Mobility Spectrometry. Journal of The American Society for Mass Spectrometry 2015, 26 (1), 14-24.

42. $\quad$ Chughtai, K.; Jiang, L.; Greenwood, T. R.; Glunde, K.; Heeren, R. M. A., Mass spectrometry images acylcarnitines, 
phosphatidylcholines, and sphingomyelin in MDA-MB-231 breast tumor models. Journal of Lipid Research 2013, 54 (2), 333-344.

43. Yan, C.; Parmeggiani, F.; Jones, E. A.; Claude, E.; Hussain, S. A.; Turner, N. J.; Flitsch, S. L.; Barran, P. E., RealTime Screening of Biocatalysts in Live Bacterial Colonies. Journal of the American Chemical Society 2017, 139 (4), 1408-1411.

44. McLean, J. A.; Ridenour, W. B.; Caprioli, R. M., Profiling and imaging of tissues by imaging ion mobility-mass spectrometry. Journal of Mass Spectrometry 2007, 42 (8), 10991105 .

45. Hart, P. J.; Francese, S.; Woodroofe, M. N.; Clench, M. R., Matrix assisted laser desorption ionisation ion mobility separation mass spectrometry imaging of ex-vivo human skin. International Journal for Ion Mobility Spectrometry 2013, 16 (2), 71-83.

46. Towers, M. W.; Karancsi, T.; Jones, E. A.; Pringle, S. D.; Claude, E., Optimised Desorption Electrospray Ionisation Mass Spectrometry Imaging (DESI-MSI) for the Analysis of Proteins/Peptides Directly from Tissue Sections on a Travelling Wave Ion Mobility Q-ToF. Journal of The American Society for Mass Spectrometry 2018, 29 (12), 2456-2466.

47. Kocurek, K. I.; Griffiths, R. L.; Cooper, H. J., Ambient ionisation mass spectrometry for in situ analysis of intact proteins. Journal of Mass Spectrometry 2018, 53 (7), 565-578.

48. $\quad$ Ridgeway, M. E.; Lubeck, M.; Jordens, J.; Mann, M.; Park, M. A., Trapped ion mobility spectrometry: A short review. International Journal of Mass Spectrometry 2018, 425, 22-35.

49. Hernandez, D. R.; DeBord, J. D.; Ridgeway, M. E.; Kaplan, D. A.; Park, M. A.; Fernandez-Lima, F., Ion dynamics in a trapped ion mobility spectrometer. Analyst 2014, 139 (8), 19131921.

50. Silveira, J. A.; Ridgeway, M. E.; Park, M. A., High Resolution Trapped Ion Mobility Spectrometery of Peptides. Analytical Chemistry 2014, 86 (12), 5624-5627.

51. Ridgeway, M. E.; Silveira, J. A.; Meier, J. E.; Park, M. A., Microheterogeneity within conformational states of ubiquitin revealed by high resolution trapped ion mobility spectrometry. Analyst 2015, 140 (20), 6964-6972.

52. Jeanne Dit Fouque, K.; Ramirez, C. E.; Lewis, R. L.; Koelmel, J. P.; Garrett, T. J.; Yost, R. A.; Fernandez-Lima, F., Effective Liquid Chromatography-Trapped Ion Mobility Spectrometry-Mass Spectrometry Separation of Isomeric Lipid Species. Analytical Chemistry 2019, 91 (8), 5021-5027.

53. Yang, J.; Caprioli, R. M., Matrix Sublimation/Recrystallization for Imaging Proteins by Mass Spectrometry at High Spatial Resolution. Analytical Chemistry 2011, 83 (14), 5728-5734.

54. Fahy, E.; Subramaniam, S.; Murphy, R. C.; Nishijima, M.; Raetz, C. R. H.; Shimizu, T.; Spener, F.; van Meer, G.; Wakelam, M. J. O.; Dennis, E. A., Update of the LIPID MAPS comprehensive classification system for lipids. Journal of Lipid Research 2009, 50 (Supplement), S9-S14.

55. Fahy, E.; Sud, M.; Cotter, D.; Subramaniam, S., LIPID MAPS online tools for lipid research. Nucleic Acids Research 2007, 35 (suppl_2), W606-W612. 